\title{
A NEW SET OF POSTULATES FOR BETWEENNESS, WITH PROOF OF COMPLETE INDEPENDENCE*
}

\author{
BY \\ EDWARD V. HUNTINGTON
}

INTRODUCTION

The paper on betweenness published by E. V. Huntington and J. R. Kline in 1917 started with a basic list of twelve postulates:

$$
\mathrm{A}, \mathrm{B}, \mathrm{C}, \mathrm{D}, 1,2,3,4,5,6,7,8 \text {, }
$$

from which eleven sets of independent postulates were selected, as follows:
(1) A, B, C, D, 1, 2;
(5) A, B, C, D, 1, 8;
(9) A, B, C, D, 3, 4, 6;
(2) A, B, C, D, 1, 5;
(6) A, B, C, D, 2, 4;
(10) A, B, C, D, 3, 4, 7;
(3) A, B, C, D, 1, 6;
(7) A, B, C, D, 2, 5;
(11) A, B, C, D, 3, 4, 8 .
(4) A, B, C, D, 1, 7;
(8) A, B, C, D, 3, 5;

Eight of these sets contain six postulates each, and three contain seven postulates each.t

In-the present paper a new postulate, called postulate 9, is added to the basic list. This new postulate leads to a twelfth set of independent postulates:

$$
\text { (12) A, B, C, D, 9, }
$$

in which the number of postulates is reduced to five. Moreover, the new postulate 9 itself is easier to remember and more convenient to handle than any of the other postulates $1-8$.

The addition of this new postulate makes desirable an extension of the discussion of the earlier paper so as to include all thirteen of the basic postulates; and this extension has been made in the present paper.

Finally, the postulates of the new set (12) are shown to be completely independent in the sense of E. H. Moore. (In regard to the other sets, a

* Presented to the Society, December 27, 1923 .

$\dagger$ E. V. Huntington and J. R. Kline, Sets of independent postulates for betweenness, these Transactions, vol. 18 (1917), pp. 301-325. 
recent paper by Mr. W. E. Van de Walle* has shown that sets (1)-(10) are completely independent, while set (11) is not.)

It is hoped that the material now available on the simple relation of "betweenness," including as it does, 12 sets of postulates with the "complete existential theory" of each set, and no less than 200 demonstrated theorems (116 on deducibility and 84 on non-deducibility), may prove of special interest to students of logic, since it provides the most elaborate known example of an abstract deductive theory.

\section{The basic List of thiRTeen postulates}

The universe of discourse consists of all systems $\mathrm{K}, \mathrm{R}$, where $\mathrm{K}$ is a class of elements, $A, B, C, \ldots$, and $\mathrm{R}(A B C)$ is a triadic relation; among these systems $(K, R)$ we designate as "betweenness" systems those that satisfy the following thirteen conditions, or postulates.

Postulate A. $A B C . \supset . C B A$.

(That is, if $A B C$ is true, then $C B A$ is true.)

Postulate B. $A \neq B . B \neq C . C \neq A: \supset: B A C-C A B-A B C \smile C B A-$ $A C B-B C A$.

(That is, if $A, B, C$ are distinct, then at least one of the six possible permutations will form a true triad.)

Postulate C. $A \neq X . X \neq Y . Y \neq A: \supset: A X Y . A Y X .=.0$.

(That is, if $A, X, Y$ are distinct, then we cannot have $A X Y$ and $A Y X$ both true at the same time.)

Postulate D. $A B C: \supset: A \neq B . B \neq C . C \neq A$.

(That is, if $A B C$ is true, then the elements $A, B$, and $C$ are distinct.)

Postulates 1-8. If $A, B, X, Y$, are distinct, then:

1. $X A B \cdot A B Y$. כ.XAY.

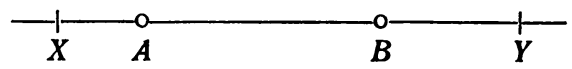

2. $X A B \cdot A Y B \cdot \supset \cdot X A Y$.

3. $X A B \cdot A Y B$ כ.XYB.

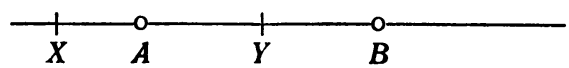

4. $A X B \cdot A Y B \cdot \supset \cdot A X Y \backsim A Y X$.

5. $A X B \cdot A Y B \cdot \supset \cdot A X Y-Y X B$.

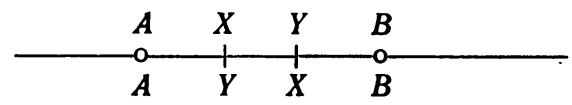

6. $X A B . Y A B$. ว. $X Y A-Y X B$.

7. $X A B \cdot Y A B \cdot \supset . X Y A-Y X A$.

8. $X A B . Y A B \cdot \supset . X Y A-Y X B$.

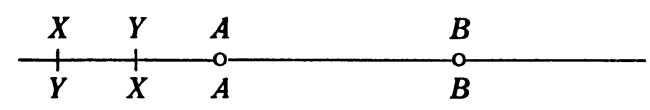

-W. E. Van de Walle, On the complete independence of the postulates for betweenness, in the present number of these Transactions, pp. 249-256. 
Postulate 9. If $A, B, C, X$ are distinct, then $A B C . X$. . . $A B X-X B C$. The new postulate 9 may be read as follows: If $A B C$ is true, and if $X$ is any fourth element distinct from $A$ and $B$ and $C$, then $X$ must lie either on the right of the middle element (giving $A B X$ ), or else on the left of the middle element (giving $X B C$ ).

In regard to certain peculiarities of postulates 5 and 8 , see under Theorem 5k, below.

\section{THEOREMS ON DEDUCIBILITY}

Besides the 71 theorems on deducibility which were stated and proved in the earlier paper, there are found to be 45 new theorems involving the new postulate 9. The proofs of these new theorems are given below, and the complete list of 116 theorems is set forth in Table $\mathrm{I}^{\prime}$.

The following proofs are supplementary to those given in the earlier paper. In each proof, the number of times that any postulate is used is indicated by an exponent.

Theorem 1e. Proof of 1 from A, C, 9.

To prove: $X A B . A B Y . \supset . X A Y$. By A, $A B Y . \supset . Y B A$. By 9, $X A B . Y$.つ. $X A Y-Y A B$. But $Y A B$ conflicts with $Y B A$, by C. Hence $X A Y$.

Theorem 2j. Proof of 2 from A, C, 9.

To prove: $X A B \cdot A Y B . \supset . X A Y$. By A, $A Y B \cdot \supset . B Y A$. By 9, $X A B . Y . \supset . X A Y-Y A B$. But if $Y A B$, then by A, $B A Y$, which conflicts with $B Y A$, by C. Hence $X A Y$.

Theorem 3f. Proof of 3 from $\mathrm{A}, \mathrm{C}^{2}, 9^{2}$.

To prove: $X A B . A Y B . \supset . X Y B$. Suppose $X Y B$ is false. First, by 9 , $A Y B . X . \supset . A Y X-X Y B$; hence $A Y X$, whence by A, $X Y A$. Second, by $9, X A B, Y . \supset . X A Y-Y A B$; but $Y A B$ conflicts with $A Y B$, by A and $\mathrm{C}$; hence $X A Y$. But thirdly, $X Y A$ and $X A Y$ conflict with each other, by $C$. Therefore $X Y B$ must be true.

Theorem 3g. Proof of 3 from A, $1^{2}, 9^{2}$.

To prove: $X A B \cdot A Y B . \supset . X Y B$. Suppose $X Y B$ is false. By 9, $A Y B . X . \supset . A Y X-X Y B$; hence $A Y X$, whence, by A, $X Y A$. By 9, $X A B . Y \cdot \supset . X A Y-Y A B$.

Case 1. If $Y A B$, then by $1, X Y A, Y A B . \supset . X Y B$.

Case 2. If $X A Y$, then by $\mathrm{A}$ and $1, B Y A . Y A X . \supset . B Y X$, whence, by $\mathrm{A}, X Y B$. 
Theorem 3h. Proof of 3 from A, B, $2^{4}, 9$.

To prove: $X A B \cdot A Y B . \supset . X Y B$. Suppose $X Y B$ is false. Then, by $B$ and $A, Y X B-X B Y$.

Case 1. If $Y X B$, then by $2, Y X B . X A B . \supset . Y X A$; hence, by 2 and $A$, $B Y A . Y X A$. ว. $B Y X$, whence, by A, $X Y B$.

Case 2. If $X B Y$, then by 2 and $A, Y B X . B A X . \supset . Y B A$.

Now by $9, A Y B . X . \supset . A Y X-X Y B$; but $X Y B$ is false; hence $A Y X$; whence, by A, XYA. Then by $2, X Y A, Y B A . \supset . X Y B$.

Theorem 3i. Proof of 3 from A, $2^{3}, 6,9$.

To prove: $X A B \cdot A Y B . \supset . X Y B$. Suppose $X Y B$ is false. By 9, $A Y B . X . \supset . A Y X-X Y B$; hence $A Y X$. Then by A and $2, B A X . A Y X . \supset . B A Y$, whence, by A, $Y A B$. Then by $6, X A B . Y A B . \supset . X Y B-Y X B$; but $X Y B$ is false; hence $Y X B$. Then by $2, Y X B . X A B . \supset . Y X A$. Hence, by $A$ and $2, B Y A . Y X A . \supset . B Y X$, whence, by $\mathrm{A}, X Y B$.

Theorem 3j. Proof of 3 from A, $2^{4}, 7,9$.

To prove: $X A B \cdot A Y B \cdot \supset . X Y B$. Suppose $X Y B$ is false. By 9, $A Y B . X . \supset . A Y X-X Y B$; hence $A Y X$, whence, by $\mathrm{A}, X Y A$. Then by $\mathrm{A}$ and 7, $B Y A . X Y A . \supset . B X Y-X B Y$.

Case 1. If $B X Y$, then by $\mathrm{A}$ and $2, Y X B . X A B . \supset . Y X A$. Then by $\mathrm{A}$ and $2, B Y A . Y X A . \supset . B Y X$, whence by A, $X Y B$.

Case 2. If $X B Y$, then by $A$ and $2, Y B X . B A X . \supset . Y B A$. Then by 2, $X Y A . Y B A . \supset . X Y B$.

Theorem 3k. Proof of 3 from A, 22, 8, 9.

To prove: $X A B, A Y B . \supset . X Y B$. Suppose $X Y B$ is false. By 9, $A Y B . X . \supset . A Y X-X Y B$; hence $A Y X$, whence by A, $X Y A$. By 2, $X A B \cdot A Y B \cdot \supset . X A Y$, whence by A, $Y A X$. Then by $\mathrm{A}$ and 8 , $Y A X . B A X . \supset . Y B A-B Y X$, whence by A, $Y B A-X Y B$; but $X Y B$ is false; hence $Y B A$. Then by 2, XYA.YBA.つ.XYB.

Theorem 4 k. Proof of 4 from A, C, $9^{2}$.

To prove: $A X B \cdot A Y B \cdot \supset . A X Y-A Y X$. Suppose both $A X Y$ and $A Y X$ are false.

By 9, $A X B . Y . \supset . A X Y-Y X B$; hence $Y X B$.

By $9, A Y B . X . \supset . A Y X-X Y B$; hence $X Y B$.

But $Y X B$ and $X Y B$ conflict with each other, by $\mathrm{A}$ and $\mathrm{C}$. Hence $A X Y \smile A Y X$.

Theorem 4l. Proof of 4 from A, $1^{2}, 7^{2}, 9$.

To prove: $A X B \cdot A Y B . \supset . A X Y-A Y X$. Suppose both $A X Y$ and $A Y X$ are false. By $9, A X B . Y . \supset . A X Y-Y X B$; hence $Y X B$. 
Then by 7, $A X B, Y X B \cdot \supset . A Y X-Y A X$; hence $Y A X$.

By $1, Y A X . A X B . \supset . Y A B$.

By 1 and $\mathrm{A}, X A Y . A Y B . \supset . X A B$.

Then by 7, $Y A B . X A B . \supset . Y X A-X Y A$. Hence by A, $A X Y-A Y X$.

Theorem $4 \mathrm{~m}$. Proof of 4 from $\mathrm{A}, 3^{2}, 7^{2}, 9^{2}$.

To prove: $A X B \cdot A Y B . \supset . A X Y-A Y X$. Suppose both $A X Y$ and $A Y X$ are false.

By 9, $A X B, Y . \supset . A X Y-Y X B$. Hence $Y X B$, whence, by A, $B X Y$.

By 9, $A Y B . X . \supset . A Y X-X Y B$. Hence $X Y B$, whence, by A, $B Y X$.

Then by 7, $A X B . Y X B$. . $A Y X-Y A X$; hence $Y A X$, whence, by A, $X A Y$.

By $3, B Y X . Y A X . \supset . B A X$; and by $3, B X Y . X A Y . \supset . B A Y$.

Then by 7 and $\mathrm{A}, Y A B . X A B . \supset . Y X A-X Y A$, whence, by A, $A X Y \smile A Y X$.

Theorem $4 \mathrm{n}$. Proof of 4 from A, 7, 82, $9^{2}$.

To prove: $A X B \cdot A Y B \cdot \supset \cdot A X Y-A Y X$. Suppose both $A X Y$ and $A Y X$ are false.

By 9, $A X B . Y . \supset . A X Y-Y X B$; hence $Y X B$. By 9, $A Y B . X$.つ. $A Y X-X Y B$; hence $X Y B$.

Then by $8, A X B . Y X B . \supset . A Y X-Y A B$; hence $Y A B$.

Also, by 8, $A Y B . X Y B . \supset . A X Y-X A B$; hence $X A B$.

Then by 7, YAB.XAB.つ.YXA-XYA, whence, by A, $A X Y \smile A Y X$.

Theorem 40. Proof of 4 from $\mathrm{C}^{2}, 7^{3}, 8^{2}, 9^{2}$.

To prove: $A X B \cdot A Y B . \supset . A X Y \smile A Y X$. Suppose both $A X Y$ and $A Y X$ are false.

By 9, $A X B, Y . \supset . A X Y-Y X B$; hence $Y X B$.

By $9, A Y B . X . \supset . A Y X-X Y B$; hence $X Y B$.

Then by 7, $A X B . Y X B . \supset . A Y X-Y A X$; hence $Y A X$. And by 7, $A Y B \cdot X Y B . \supset . A X Y-X A Y$; hence $X A Y$.

Also by $8, A X B . Y X B \cdot \supset . A Y X-Y A B$; hence $Y A B$ and by 8 , $A Y B \cdot X Y B . \supset \cdot A X Y \smile X A B$; hence $X A B$.

Then by 7, YAB.XAB.つ.YXA-XYA.

But $Y X A$ conflicts with $Y A X$, by $\mathrm{C}$, and $X Y A$ conflicts with $X A Y$, by $\mathrm{C}$.

Therefore $A X Y-A Y X$.

Theorem 4p. Proof of 4 from $2^{2}, 9^{2}$.

To prove: $A X B \cdot A Y B \cdot \supset \cdot A X Y \smile A Y X$. Suppose both $A X Y$ and $A Y X$ are false.

By 9, $A X B . Y . \supset . A X Y-Y X B$; hence $Y X B$.

By $9, A Y B . X . \supset . A Y X-X Y B$; hence $X Y B$. 
Then by $2, A Y B . Y X B \cdot \supset . A Y X$, and by 2, $A X B \cdot X Y B . \supset . A X Y$.

Therefore $A X Y-A Y X$.

Theorem 5k. Proof of 5 from 9.

To prove: $A X B \cdot A Y B . \supset . A X Y-Y X B$.

By 9, $A X B . Y . \supset . A X Y-Y X B$; which was to be proved. It will be observed that only the first part of the hypothesis is used in the proof. Postulate 9 is "stronger" than postulate 5. By interchanging $X$ and $Y$, postulate 5 may also be written in the form

$$
A Y B \cdot A X B \cdot \supset \cdot A Y X-X Y B
$$

which may be proved as follows: By 9 ,

$$
A Y B . X . \supset . A Y X-X Y B
$$

which was to be proved. Here again, only the first part of the hypothesis is used in the proof.

Furthermore, since " $A X B$ and $A Y B$ " is logically equivalent to " $A Y B$ and $A X B$ ", postulate 5 may be written in either of the following forms:

$$
\begin{aligned}
& A X B \cdot A Y B \cdot \text { ว. } A Y X-X Y B . \\
& A Y B \cdot A X B \cdot \text {. } A X Y-Y X B .
\end{aligned}
$$

It is interesting to notice, however, that no one of these four forms is a significant statement, unless one part of the hypothesis is recognized specifically as the "first part" and the other as the "second part" - a distinction which, strictly speaking, introduces a foreign element into the statement of the proposition.

In order to avoid the necessity of making this arbitrary distinction between the "first" and the "second" term of a pair connected by a simple "and", we may restate postulate 5 in the following less objectionable form:

$$
\text { 5'. } A X B . A Y B: \supset:(A X Y-Y X B) .(A Y X-X Y B) .
$$

This should not be regarded as merely a combination of two of the separate statements mentioned above, since, without employing the distinction between "first" and "second", we cannot tell which part of the hypothesis is supposed to be paired with which part of the conclusion. It is only when the statement $\left(5^{\prime}\right)$ is taken as a whole that it can be translated into significant words, without using the distinction between the "first" and "second" parts of the simple conjunction which forms the hypothesis. 
Thus, $5^{\prime}$ may be read as follows: "The two triads in the hypothesis contain the same initial element, $A$, and the same terminal element, $B$, but different middle elements, $X$ and $Y$ (which we may call the "odd elements"). The conclusion also consists of two parts. One part says that at least one of the following triads is true:

(A) (one odd) (the other odd) or (the other odd) (the one odd) $(B)$; the other part says that at least one of the following is true:

(A) (the other odd) (the one odd) or (the one odd) (the other odd) (B)."

Now neither of these parts alone gives us any definite information unless we are able to recognize the "one" as $X$ and the "other" as $Y$ (or vice versa); but the two parts together give an unequivocal conclusion whether the "one" $=X$ and the "other" $=Y$, or the "one" $=Y$ and the "other" $=X$.

Precisely the same remarks apply to pọstulate 8 , which may be re-stated more strictly as follows:

$$
\text { 8'. } X A B . Y A B: \supset:(X Y A-Y X B) .(Y X A-X Y B) .
$$

Fortunately, these logical refinements do not affect the essential reasoning, provided the precaution already stated in the footnote on page 318 of the earlier paper is observed.

Theorem 6k. Proof of 6 from A, B, C 29.

To prove: $X A Y . Y A B . \supset . X Y B-Y X B$. By B and A, $X Y B-Y X B-X B Y$. Suppose $X B Y$. Then by $9, X B Y . A . \supset . X B A-A B Y$. But $X B A$ conflicts with $X A B$, by $\mathrm{C}$; and $A B Y . \supset . Y B A$, by A, which conflicts with $Y A B$, by C. Hence $X Y B-Y X B$.

Theorem 61. Proof of 6 from A, C, 7, 9.

To prove: $X A B . Y A B . \supset . X Y B-Y X B$.

By 7, XAB,YAB.つ.XYA-YXA.

Case 1 . If $X Y A$, then by $9, X Y A . B . \supset . X Y B-B Y A$. But $B Y A$ conflicts with $Y A B$, by $\mathrm{C}$ and $\mathrm{A}$. Hence, in Case $1, X Y B$.

Case 2. If $Y X A$, then by $9, Y X A . B . \supset . Y X B-B X A$. But $B X A$ conflicts with $X A B$, by $\mathrm{C}$ and $\mathrm{A}$. Hence, in Case 2, $Y X B$.

THEOREM $6 \mathrm{~m}$. Proof of 6 from A, $2^{4}, 7^{3}, 9^{2}$.

To prove: $X A B, Y A B . \supset . X Y B \sim Y X B$. Suppose both $X Y B$ and $Y X B$ (and hence, by A, also $B Y X$ and $B X Y$ ) are false.

By 7, $X A B . Y A B$. つ.XYA-YXA.

Case 1. If $X Y A$, then by $9, X Y A . B . \supset . X Y B-B Y A$. But $X Y B$ is false. Hence $B Y A$, and by A, $A Y B$. Then by 7, $B Y A . X Y A . \supset . B X Y \smile X B Y$. 
But $B X Y$ is false. Hence $X B Y$. Then by 2 and A, $Y B X . B A X . \supset . Y B A$. Hence by 2, XYA.YBA.?.XYB.

Case 2. If $Y X A$, then by $9, Y X A \cdot B . \supset . Y X B \cdot B X A$. But $Y X B$ is false. Hence $B X A$. Then by 7 and $\mathrm{A}, B X A . Y X A . \supset . B Y X-Y B X$. But $B Y X$ is false. Hence $Y B X$. Then by 2 and $\mathrm{A}, X B Y . B A Y . \supset . X B A$. Hence by 2, YXA.XBA.つ.YXB.

Therefore $X Y B-Y X B$.

Theorem 6n. Proof of 6 from A, 2, $8^{3}, 9$.

To prove: $X A B . Y A B . \supset . X Y B-Y X B$. Suppose both $X Y B$ and $Y X B$ are false. Then, by $\mathrm{A}$, both $B Y X$ and $B X Y$ are false.

By 8, XAB.YAB.つ.XYA $Y X B$; hence $X Y A$.

By 8, YAB.XAB.つ. $Y X A-X Y B$; hence $Y X A$.

By 9, XYA.B.つ.XYB-BYA; hence $B Y A$.

By $8, B Y A . X Y A . \supset . B X Y-X B A$; hence $X B A$.

By 2, $Y X A . X B A . \supset . Y X B$ contrary to supposition.

Theorem 60. Proof of 6 from A, 4, $8^{2}, 9^{2}$.

To prove: $X A B . Y A B . \supset . X Y B-Y X B$. Suppose both $X Y B$ and $Y X B$ are false.

By 8, $X A B . Y A B$. . . XYA $-Y X B$; hence $X Y A$, and by A, $A Y X$.

By 8, $Y A B . X A B . \supset . Y X A-X Y B$; hence $Y X A$, and by A, $A X Y$.

By 9, $X Y A . B . \supset . X Y B-B Y A$; hence $B Y A$.

By 9, YXA.B.つ. YXB-BXA; hence $B X A$.

Then by $4, B X A . B Y A . \supset . B X Y-B Y X$. Hence, by A, $X Y B-B X Y$.

Theorem 6 p. Proof of 6 from A, 7, $8^{4}, 9^{2}$.

To prove: $X A B . Y A B$. $. X Y B-Y X B$. Suppose both $X Y B$ and $Y X B$ are false. Then by $\mathrm{A}, B Y X$ and $B X Y$ are false.

By 8, $X A B . Y A B . \supset . X Y A-Y X B$; hence $X Y A$.

By 8, YAB.XAB.つ. $Y X A-X Y B$; hence $Y X A$.

By 9, $X Y A . B$. . $X Y B-B Y A$; hence $B Y A$.

By 9, YXA.B.つ. YXB-BXA; hence $B X A$.

By 8, $B Y A . X Y A . \supset . B X Y \smile X B A$; hence $X B A$.

By 8, $B X A . Y X A . \supset . B Y X-Y B A$; hence $Y B A$.

Then by 7, $X B A . Y B A$. ๖. $X Y B-Y X B$.

Theorem $6 \mathrm{q}$. Proof of 6 from $\mathrm{C}^{3}, 8^{4}, 9^{2}$.

To prove: $X A B . Y A B . \supset . X Y B-Y X B$. Suppose both $X Y B$ and $Y X B$ are false.

By 8, XAB.YAB.つ.XYA $Y X B$; hence $X Y A$. 
By 8, YAB.XAB.つ. YXA-XYB; hence $Y X A$.

By 9, XYA.B.つ.XYB-BYA; hence $B Y A$.

By 9, $Y X A . B$. . $Y X B-B X A$; hence $B X A$.

By 8, $B Y A . X Y A$. . $B X Y-X B A$. But $X B A$ conflicts with $X A B$ by C. Hence $B X Y$.

By 8, $B X A . Y X A$. כ. $B Y X-Y B A$. But $Y B A$ conflicts with $Y A B$ by C. Hence $B Y X$.

Now $B X Y$ and $B Y X$ conflict with each other, by $\mathrm{C}$.

Therefore $X Y B \smile Y X B$.

Theorem $7 \mathrm{k}$. Proof of 7 from A, B, C $\mathrm{C}^{4}, 9^{3}$.

To prove: $X A B . Y A B . \supset . X Y A-Y X A$.

By B, $X Y B-Y X B-X B Y$.

Case 1. If $X Y B$, then by 9, XYB.A.つ.XYA-AYB. But $A Y B$ conflicts with $B A Y$, by $\mathrm{C}$ and $\mathrm{A}$. Hence in Case $1, X Y A$.

Case 2. If $Y X B$, then by $9, Y X B . A . \supset . Y X A-A X B$. But $A X B$ conflicts with $X A B$, by $\mathrm{C}$ and $\mathrm{A}$. Hence in Case 2, YXA.

Case 3. Suppose $X B Y$. Then by 9, $X B Y . A . \supset . X B A-A B Y$. But $X B A$ conflicts with $X A B$, by $\mathrm{C}$; and $A B Y$ conflicts with $Y A B$, by $\mathrm{C}$ and $A$. Hence Case 3 is impossible.

Therefore $X Y A-Y X A$.

Theorem 7l. Proof of 7 from A, C $\mathrm{C}^{2}, 6,9^{2}$.

To prove: $X A B . Y A B . \supset . X Y A-Y X A$.

By 6, XAB.YAB.つ.XYB-YXB.

Case 1. If $X Y B$, then by 9, XYB.A.つ.XYA-AYB. But $A Y B$ conflicts with $Y A B$, by $\mathrm{C}$ and $\mathrm{A}$. Hence, in Case $1, X Y A$.

Case 2. If $Y X B$, then by $9, Y X B \cdot A . \supset Y X A-A X B$. But $A X B$ conflicts with $X A B$, by $\mathrm{C}$ and $\mathrm{A}$. Hence, in Case $2, Y X A$.

Therefore $X Y A-Y X A$.

Theorem $7 \mathrm{~m}$. Proof of 7 from A, 4, 82, $9^{2}$.

To prove: $X A B . Y A B . \supset . X Y A-Y X A$. Suppose both $X Y A$ and $Y X A$ are false.

By 8, XAB.YAB.?.XYA-YXB; hence $Y X B$.

By 8, YAB.XAB. . $Y X A-X Y B$; hence $X Y B$.

Then by $9, Y X B . A$. ?. $Y X A-A X B$; hence $A X B$.

And by 9, $X Y B . A$. כ.XYA-AYB; hence $A Y B$.

Then by 4, $A X B, A Y B . \supset . A X Y \smile A Y X$. Hence by A, $X Y A \smile Y X A$.

Theorem 7n. Proof of 7 from A, $1^{2}, 4^{3}, 6^{3}, 9^{2}$.

To prove: $X A B . Y A B . \supset . X Y A-Y X A$. Suppose both $X Y A$ and $Y X A$ are false. Then by A, $A Y X$ and $A X Y$ are false. 
By 6, XAB.YAB.つ.XYB-YXB.

Case 1. If $X Y B$ is true and $Y X B$ false, then by 4, $X A B . X Y B$.つ.XAY $X Y A$; hence $X A Y$. Then by 6 and $\mathrm{A}, X A Y . B A Y$. . .XBY $B X Y$, whence, by $\mathrm{A}$, $Y B X-Y X B$. But $Y X B$ is false, hence $Y B X$. Now by 9, $X Y B . A . \supset . X Y A-A Y B$; hence $A Y B$. Then by $1, A Y B, Y B X . \supset . A Y X$, which is false.

Case 2. If $Y X B$ is true and $X Y B$ false, then by 4, $Y X B$. $Y A B$.つ. $Y X A-Y A X$; hence $Y A X$. Then by 6 and $A, Y A X . B A X$. ? . $Y B X-B Y X$, whence, by A, $X B Y-X Y B$. But $X Y B$ is false; hence $X B Y$. By 9, $Y X B . A$. . $Y X A-A X B$; hence $A X B$. Then by $1, A X B . X B Y . \supset . A X Y$, which is false.

Case 3. If $X Y B$ and $Y X B$ are both true, then by 9, $X Y B . A$. . . $X Y A \smile A Y B$; hence $A Y B$. And by $9, Y X B . A . \supset . Y X A-A X B$; hence $A X B$. Then by 4, $A X B \cdot A Y B . \supset . A X Y-A Y X$. Therefore by A, $X Y A-Y X A$.

Theorem 8n. Proof of 8 from A, B, C $\mathrm{C}^{3}, 9^{2}$.

To prove: $X A B . Y A B . \supset . X Y A-Y X$ B. By B, $X B Y-X Y B-Y X B$.

Case 1. Suppose $X B Y$; then by 9, $X B Y . A . \supset . X B A-A B Y$. But $X B A$ conflicts with $X A B$, by $\mathrm{C}$; and $A B Y$ conflicts with $Y A B$, by $\mathbf{A}$ and $\mathbf{C}$.

Case 2. If $X Y B$, then by 9, XYB.A. . $X Y A-A Y B$. But $A Y B$ conflicts with $B A Y$, by $\mathrm{A}$ and $\mathrm{C}$.

Therefore $X Y A-Y X B$.

Theorem 80. Proof of 8 from A, B, $1^{2}, 6^{2}, 9$.

To prove: $X A B . Y A B . \supset . X Y A-Y X B$. Suppose both $X Y A$ and $Y X B$ are false. By 6, $X A B . Y A B . \supset . X Y B-Y X B$; hence $X Y B$. Then by 9, $X Y B . A . \supset . X Y A-A Y B$; hence $A Y B$.

By $\mathrm{B}$ and $\mathrm{A}, Y X A-X A Y-X Y A$; hence $Y X A$ or $X A Y$. But if $Y X A$, then by $1, Y X A, X A B . \supset . Y X B$, which is false; hence $X A Y$. Then by 6 and $\mathrm{A}, X A Y . B A Y$. . . XBY $-B X Y$. But if $B X Y$, then by $\mathrm{A}, Y X B$, which is false; hence $X B Y$, whence, by A, $Y B X$. Then by $1, A Y B . Y B X . \supset . A Y X$, whence, by $\mathrm{A}, X Y A$, which is false.

Therefore $X Y A-Y X B$.

Theorem 8p. Proof of 8 from A, C, 6, 9 .

To prove: $X A B . Y A B . \supset . X Y A-Y X B$. Suppose both $X Y A$ and $Y X B$ are false. By 6, $X A B . Y A B$. . . XYB $-Y X B$; hence $X Y B$. Then by 9, $X Y B . A$. ๖.XYA-AYB. But $A Y B$ conflicts with $Y A B$ by $\mathrm{C}$ and $\mathrm{A}$; and $X Y A$ is false.

Therefore $X Y A-Y X B$.

Theorem 8q. Proof of 8 from A, C, 7, 9.

To prove: $X A B . Y A B$. . $X Y A-Y X B$. We may vary the method of proof, as follows: By 7, XAB.YAB.つ.XYA-YXA. If $X Y A$, the 
theorem is established. Suppose $Y X A$; then by $9, Y X A . B . \supset . Y X B-B X A$. If $Y X B$, the theorem is established. Suppose $B X A$. By A, $X A B$.つ. $B A X$, which conflicts with $B X A$, by $\mathrm{C}$. Therefore the theorem must be true.

Theorem 8r. Proof of 8 from A, 1, 4, 6², 9 .

To prove: $X A B . Y A B$. . . XYA $-Y X B$. Suppose both $X Y A$ and $Y X B$ are false. By 6, $X A B . Y A B . \supset . X Y B-Y X B$; hence $X Y B$. Then by 4, $X Y B . X A B . \supset . X Y A-X A Y$; hence $X A Y$; and by 9, XYB.A.つ.XYA-AYB; hence $A Y B$.

By $\mathrm{A}$ and $6, B A Y . X A Y . \supset . B X Y-X B Y$, whence, by A, $Y X B-Y B X$; hence $Y B X$. Then by $1, A Y B . Y B X . \supset . A Y X$, whence, by A, $X Y A$, which is false.

Therefore $X Y A-Y X B$.

Theorem 8s. Proof of 8 from A, $2^{3}, 7^{2}, 9$.

To prove: $X A B . Y A B$. . $X Y A-Y X B$. Suppose both $X Y A$ and $Y X B$ are false. By 7, $X A B . Y A B . \supset . X Y A-Y X A$; hence $Y X A$. Then by 9 , $Y X A . B . \supset . Y X B-B X A$; hence $B X A$. Then by 7, $Y X A . B X A . \supset . Y B X-B Y X$.

Case 1. If $Y B X$, then by $\mathrm{A}$ and $2, X B Y . B A Y . \supset . X B A$. Then by 2, $Y X A . X B A \cdot$. $Y X B$.

Case 2. If $B Y X$, then by $\mathrm{A}$ and $2, X Y B . Y A B . \supset . X Y A$.

Therefore $X Y A-Y X B$.

Theorem 9a. Proof of 9 from A, B, 1, 2.

To prove: $A B C . X$.つ. $A B X-X B C$. By B and $\mathrm{A}$ we have $X B C-B C X-B X C$. If $B C X$, then $A B C \cdot B C X$.つ. $A B X$, by 1. If $B X C$, then $A B C . B X C$. . $A B X$, by 2. Hence $A B X-X B C$.

Theorem 9b. Proof of 9 from A, B $\mathrm{B}^{3}, \mathrm{C}^{\mathbf{4}}, \mathbf{5}$.

To prove: $A B C . X . \supset . A B X-X B C$. Suppose both $A B X$ and $X B C$ are false. Then, by $\mathrm{B}$ and $\mathrm{A}, A X B-B A X$ and by $\mathrm{B}$ and $\mathrm{A}, B X C-B C X$.

Case 1. If $B C X$, then by $1, A B C . B C X . \supset . A B X$.

Case 2. If $B A X$, then by 1 and $\mathrm{A}, C B A . B A X . \supset . C B X$, whence, by $\mathrm{A}, X B C$.

Case 3. If $B X C$ and $A X B$, use B again: $A C X-C A X-C X A$.

Suppose $A C X$. Then by 1 and $\mathrm{A}, A C X . C X B$. つ. $A C B$, contrary to $A B C$, by $\mathrm{C}$.

Suppose $C A X$. Then by $1, C A X . A X B . \supset . C A B$, contrary to $A B C$, by $\mathrm{C}$ and $\mathrm{A}$.

Suppose $C X A$. Then by 5 and A, $C B A . C X A . \supset . C B X-X B A$. Hence, by $\mathrm{A}, A B X-X B C$. 
Theorem $9 \mathrm{c}$. Proof of 9 from $\mathrm{A}, \mathrm{B}^{2}, \mathrm{C}^{4}, 1^{2}, 6^{2}$.

To prove: $A B C . X . \supset . A B X-X B C$.

Suppose both $A B X$ and $X B C$ are false. Then, by B and A, $A X B-B A X$ and, by $\mathrm{B}$ and $\mathrm{A}, B X C-B C X$.

Case 1. If $B A X$ and $B X C$, then by $\mathrm{A}$ and 1, $X A B . A B C . \supset . X A C$, whence by $A$ and $6, B A X . C A X$. つ. $B C X-C B X$; but both $B C X$ and $C B X$ conflict with $B X C$, by $\mathrm{C}$ and $\mathrm{A}$.

Case 2. If $A X B$ and $B X C$, then, by $\mathrm{A}$ and 6, $A X B . C X B . \supset . A C B-C A B$, both of which conflict with $A B C$, by $\mathrm{C}$ and $\mathrm{A}$.

Case 3. If $B C X$, then, by $1, A B C \cdot B C X . \supset . A B X$, which is false.

Therefore $A B X \smile X B C$.

Theorem $9 \mathrm{~d}$. Proof of 9 from $\mathrm{A}, \mathrm{B}^{2}, \mathrm{C}^{2}, 1^{4}, 7$.

To prove: $A B C . X . \supset . A B X-X B C$. Suppose both $A B X$ and $X B C$ are false. Then, by $\mathrm{B}$ and $\mathrm{A}, B A X-A X B$, and, by $\mathrm{B}$ and $\mathrm{A}, B C X-C X B$.

Case 1. If $B A X$, then by 1 and $A, C B A . B A X . \supset . C B X$, whence $X B C$, by $\mathrm{A}$.

Case 2. If $B C X$, then by $1, A B C . B C X . \supset . A B X$.

Case 3. If $C X B$ and $A X B$, then by 7, $C X B . A X B . \supset . C A X-A C X$. But if $C A X$, then by $1, C A X . A X B . \supset . C A B$, and if $A C X$, then by 1 , $A C X . C X B$. つ. $A C B$, both of which conflict with $A B C$, by $\mathrm{C}$.

Therefore $A B X-X B C$.

Theorem 9e. Proof of 9 from $\mathrm{A}, \mathrm{B}^{2}, \mathrm{C}^{3}, 1^{2}, 8^{2}$.

To prove: $A B C . X . \supset . A B X-X B C$. Suppose both $A B X$ and $X B C$ were false. Then, by $\mathrm{B}$ and $\mathrm{A}, A X B-B A X$, and, by B and A, $C X B-B C X$.

Case 1. If $B C X$, then, by $1, A B C \cdot B C X . \supset . A B X$.

Case 2. If $B A X$, then, by 1 und $A, C B A \cdot B A X . \supset . C B X$, whence, by A, $X B C$.

Case 3. Suppose $A X B$ and $C X B$, then, by 8, $A X B . C X B$. ว. $A C X-C A B$ and, by 8,CXB.AXB.つ.CAX $-A C B$. But $A C X$ and $C A X$ conflict with each other, by $\mathrm{A}$ and $\mathrm{C}$; $C A B$ conflicts with $A B C$, by $\mathrm{A}$ and $\mathrm{C}$; and $A C B$ conflicts with $A B C$, by $\mathrm{C}$. Hence Case 3 is impossible.

Therefore $A B X-X B C$.

'Theorem 9f. Proof of 9 from A, B ${ }^{2}, \mathrm{C}^{2}, 2^{2}, 4$.

To prove: $A B C . X . \supset . A B X-X B C$. Suppose both $A B X$ and $X B C$ were false. Then, by $\mathrm{B}$ and $\mathrm{A}, B X A-B A X$, and, by $\mathrm{B}$ and $\mathrm{A}, B X C-B C X$.

Case 1. If $B X C$, then, by $2, A B C . B X C \cdot \supset . A B X$.

Case 2. If $B X A$, then, by 2 and $\mathrm{A}, C B A . B X A . \supset . C B X$, whence, by $\mathrm{A}, X B C$. 
Case 3. If $B A X$ and $B C X$, then, by 4, $B A X . B C X . \supset . B A C-B C A$, both of which conflict with $A B C$, by $\mathrm{C}$.

Hence $A B X \smile X B C$.

Theorem 9g. Proof of 9 from $\mathrm{A}, \mathrm{B}^{2}, \mathrm{C}^{3}, 2^{2}, 5^{2}$.

To prove: $A B C . X . \supset . A B X-X B C$. Suppose both $A B X$ and $X B C$ were false. Then, by $\mathrm{B}$ and $\mathrm{A}, B A X-B X A$ and $B C X-B X C$.

Case 1. If $B X C$, then, by 2, $A B C . B X C . \supset . A B X$.

Case 2. If $B X A$, then, by 2 and $\mathrm{A}, C B A . B X A . \supset . C B X$, whence, by A, $X B C$.

Case 3. If $B A X$, and $B C X$, then, by 5, $B A X . B C X . \supset . B A C-C A X$, and, by 5, $B C X . B A X$. 2. $B C A-A C X$. But $B A C$ conflicts with $A B C$, by $\mathrm{C}$ and $\mathrm{A} ; B C A$ conflicts with $A B C$, by $\mathrm{C}$ and $\mathrm{A}$; and $C A X$ and $A C X$ conflict with each other, by $\mathrm{C}$ and $\mathrm{A}$. Hence $A B X-X B C$.

Theorem 9h. Proof of 9 from A, B, $3^{2}, 5$.

To prove: $A B C . X . \supset . A B X-X B C$. By $\mathrm{A}, C B A$. By $\mathrm{B}$ and $\mathrm{A}$, $X A C-X C A-A X C$.

If $X A C$, then, by $3, X A C . A B C . \supset . X B C$.

If $X C A$, then, by $3, X C A . C B A$. . $X B A$, whence $A B X$ by $\mathrm{A}$.

If $A X C$, then, by 5, $A B C . A X C . \supset . A B X-X B C$.

Hence, in any case, $A B X-X B C$.

Theorem 9i. Proof of 9 from $\mathrm{A}, \mathrm{B}^{2}, \mathrm{C}^{2}, 3^{2}, 4,6$.

To prove: $A B X . X . \supset . A B X-X B C$. Suppose both $A B X$ and $X B C$ were false. Then by $\mathrm{B}$ and $\mathrm{A}, B A X-A X B$ and $B C X-C X B$.

Case 1. Suppose $B A X$ and $B C X$. Then, by 4, $B A X . B C X . \supset$. $B A C-B C A$. Case 2. Suppose $B A X$ and $C X B$. Then, by 3 and $A, C X B, X A B$. . . $C A B$. Case 3. Suppose $A X B$ and $B C X$. Then, by 3 and $A, A X B . X C B$. . $A C B$.

Case 4. Suppose $A X B$ and $C X B$. Then, by 6, $A X B$. $C X B$. ? . $A C B-C A B$.

Hence, in any case, by $A, C A B-A C B$, both of which conflict with $A B C$, by $\mathrm{C}$ and $\mathrm{A}$. Therefore $A B X-X B C$.

Theorem 9j. Proof of 9 from A, B, 32, $4^{2}, 7$.

To prove: $A B C . X . \supset . A B X-X B C$.

By A, $C B A$. By B, $A X C \smile X A C \smile X C A$.

Case 1. If $A X C$, then also, by $\mathrm{A}, C X A$. Then by 4, $A X C \cdot A B C$. . $A X B-A B X$, and also, by 4,CXA.CBA. . $C X B-C B X$, whence, by $\mathrm{A}, C X B-X B C$. Then if $A B X$ and $X B C$ are both false, we must have $A X B$ and $C X B$. Hence, by 7, $C X B . A X B . \supset . C A X-A C X$, whence, by A, $X A C \smile X C A$.

Case 2. If $X A C$, then, by $3, X A C . A B C . \supset . X B C$. 
Case 3. If $X C A$, then, by $3, X C A . C B A . \supset . X B A$, whence by A, $A B X$. Therefore, in any case, $A B X-X B C$.

Theorem 9k. Proof of 9 from A, B $\mathrm{B}^{3}, 3^{2}, 4,8^{2}$.

Proof same as for Theorem 9i down to

Case 4. Suppose $A X B$ and $C X B$. Then, by 8, $A X B . C X B$. ᄀ. $A C X-C A B$, and by 8,CXB.AXB.つ.CAX $-A C B$. But $C A B$ and $A C B$ conflict with $A B C$, by $\mathrm{C}$ and $\mathrm{A}$, and $A C X$ and $C A X$ conflict with each other, by $\mathrm{C}$ and $\mathrm{A}$; so that Case 4 is impossible. Also, Cases 1,2 , and 3 conflict with $A B C$, by $\mathrm{C}$ and $\mathrm{A}$.

Hence $A B X-X B C$ must be true.

Theorem 9l. Proof of 9 from A, B, 2, $3^{2}, 4$.

To prove: $A B C . X . \supset . A B X-X B C$.

By $\mathrm{B}, X A C-X C A-A X C$.

Case 1. If $X A C$, then by $3, X A C . A B C . \supset . X B C$.

Case 2. If $X C A$, then by 3 and $\mathrm{A}, X C A . C B A . \supset . X B A$, whence, by A, $A B X$.

Case 3. If $A X C$, then by 4, $A B C . A X C . \supset . A B X-A X B$. But if $A X B$, then by 2 and $A, C B A . B X A . \supset . C B X$, whence, by A, $X B C$.

Therefore, $A B X-X B C$.

Theorem $9 \mathrm{~m}$. Proof of 9 from A, B⿳, 1, 32, 7 .

To prove: $A B C . X . \supset . A B X-X B C$. Suppose both $A B X$ and $X B C$ are false.

By B, $X A C-X C A-A X C$. But if $X A C$, then by 3, $X A C . A B C . \supset . X B C$, which is false; and if $X C A$, then by 3 and $A, X C A . C B A . \supset . X B A$, whence, by $\mathrm{A}, A B X$, which is false. Therefore $A X C$.

Now by $\mathrm{B}, X B C \backsim B C X-C X B$. But $X B C$ is false; and if $B C X$, then by $1, A B C . B C X . \supset . A B X$, which is false. Therefore $C X B$, whence, by $\mathrm{A}, B X C$.

Then by 7, $B X C . A X C \cdot \supset . B A X-A B X$. But $A B X$ is false; and if $B A X$, then by 1 and $\mathrm{A}, C B A . B A X . \supset . C B X$, whence, by $\mathrm{A}, X B C$, which is false.

Therefore $A B X-X B C$.

These 45 new theorems, together with the 71 theorems proved in the earlier paper, complete the list of $\mathbf{1 1 6}$ theorems on deducibility among the thirteen postulates of our revised basic list.

The results are collected for reference in Table $\mathrm{I}^{\prime}$. 
TABLE I'. 116 THeOREMS ON DKDUCIBILITY

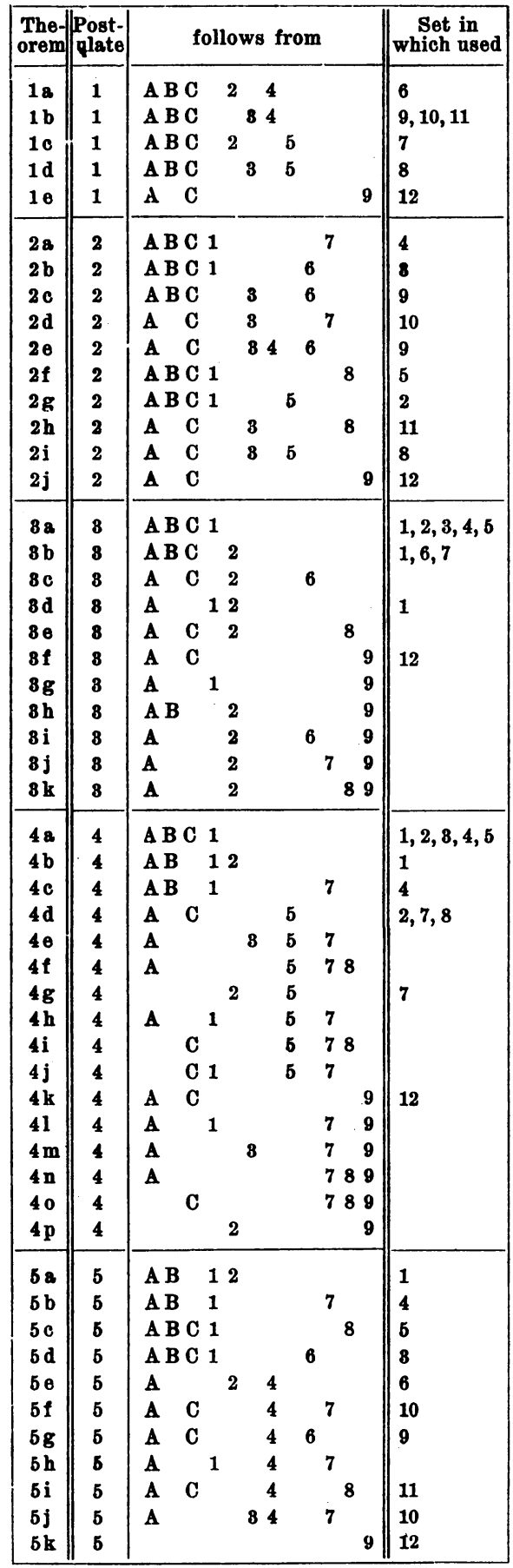

\begin{tabular}{|c|c|c|c|c|}
\hline $\begin{array}{l}\text { The- } \\
\text { orem }\end{array}$ & $\begin{array}{l}\text { Post- } \\
\text { ulate }\end{array}$ & follow & from & $\begin{array}{c}\text { Set in } \\
\text { which used }\end{array}$ \\
\hline $\begin{array}{l}6 \mathrm{a} \\
6 \mathrm{~b} \\
6 \mathrm{c} \\
6 \mathrm{~d} \\
6 \mathrm{e} \\
6 \mathrm{f} \\
6 \mathrm{~g} \\
6 \mathrm{~h} \\
6 \mathrm{i} \\
6 \mathrm{j} \\
6 \mathrm{k} \\
61 \\
6 \mathrm{~m} \\
6 \mathrm{n} \\
6 \mathrm{o} \\
6 \mathrm{p} \\
6 \mathrm{q}\end{array}$ & $\begin{array}{l}6 \\
6 \\
6 \\
6 \\
6 \\
6 \\
6 \\
6 \\
6 \\
6 \\
6 \\
6 \\
6 \\
6 \\
6 \\
6 \\
6 \\
6\end{array}$ & 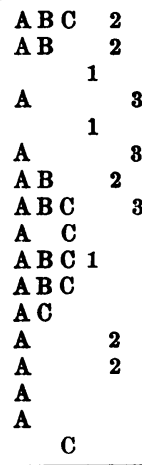 & 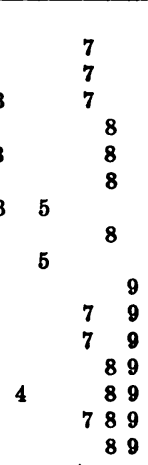 & $\begin{array}{l}1,6,7 \\
4 \\
10 \\
5 \\
11 \\
8 \\
5,11 \\
2 \\
12\end{array}$ \\
\hline $\begin{array}{l}7 \mathrm{a} \\
7 \mathrm{~b} \\
7 \mathrm{c} \\
7 \mathrm{~d} \\
7 \mathrm{e} \\
7 \mathrm{f} \\
7 \mathrm{~g} \\
7 \mathrm{~h} \\
7 \mathbf{i} \\
7 \mathbf{j} \\
\mathbf{7 k} \\
7 \mathbf{1} \\
7 \mathrm{~m} \\
7 \mathrm{n}\end{array}$ & $\begin{array}{l}7 \\
7 \\
7 \\
7 \\
7 \\
7 \\
7 \\
7 \\
7 \\
7 \\
7 \\
7 \\
7 \\
7\end{array}$ & 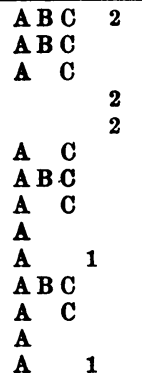 & 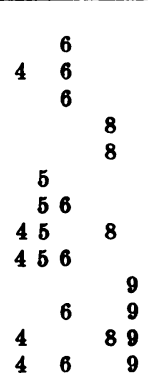 & $\begin{array}{l}1,6,7 \\
8,9 \\
9\end{array}$ \\
\hline $\begin{array}{l}8 \mathrm{ag} \\
8 \mathrm{~b} \\
8 \mathrm{c} \\
8 \mathrm{~d} \\
8 \mathrm{~g} \\
8 \mathrm{f} \\
8 \mathrm{~g} \\
8 \mathrm{~h} \\
8 \mathrm{i} \\
8 \mathrm{j} \\
8 \mathrm{k} \\
81 \\
8 \mathrm{~m} \\
8 \mathrm{n} \\
80 \\
8 \mathrm{p} \\
8 \mathrm{q} \\
8 \mathrm{r} \\
8 \mathrm{~s}\end{array}$ & $\begin{array}{l}8 \\
8 \\
8 \\
8 \\
8 \\
8 \\
8 \\
8 \\
8 \\
8 \\
8 \\
8 \\
8 \\
8 \\
8 \\
8 \\
8 \\
8 \\
8\end{array}$ & 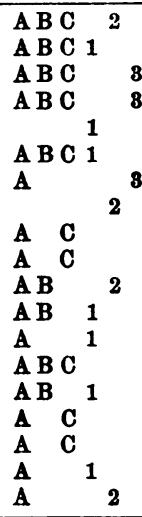 & 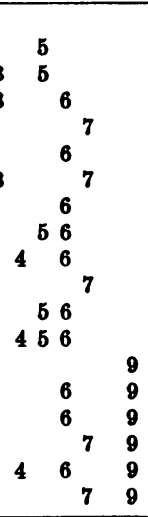 & $\begin{array}{l}1,6,7 \\
2 \\
8 \\
9 \\
4 \\
8 \\
10\end{array}$ \\
\hline $\begin{array}{l}9 \mathrm{a} \\
9 \mathrm{~b} \\
9 \mathrm{c} \\
9 \mathrm{~d} \\
9 \mathrm{e} \\
9 \mathrm{f} \\
9 \mathrm{~g} \\
9 \mathrm{~h} \\
9 \mathrm{i} \\
9 \mathrm{j} \\
9 \mathrm{k} \\
9 \mathrm{l} \\
9 \mathrm{~m}\end{array}$ & $\begin{array}{l}9 \\
9 \\
9 \\
9 \\
9 \\
9 \\
9 \\
9 \\
9 \\
9 \\
9 \\
9 \\
9\end{array}$ & 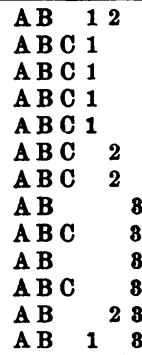 & 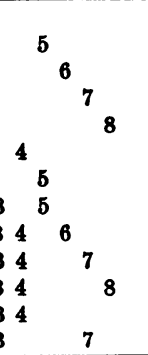 & $\begin{array}{l}1 \\
2 \\
8 \\
4 \\
5 \\
6 \\
7 \\
8 \\
9 \\
10 \\
11\end{array}$ \\
\hline
\end{tabular}


EXAMPLES OF PSEUDO-BETWEENNESS.

In order to prove that no other theorems on deducibility are possible except those stated above, we first exhibit 54 examples of pseudo-betweenness, that is, 54 examples of systems $K, R$, which have some but not all of the properties mentioned in our basic list.

Of these examples, 37 were given in the earlier paper, and 17 are new. In the table following, the numbering of the examples is so arranged as to avoid conflict with the numbering in the earlier paper. (It will be noted that seven examples of the old list, namely, 17, 22, 25, 27, 31, 34, 35, are now omitted, being no longer needed, in view of certain of the new examples.)

In the case of each example, the postulates which are satisfied are mentioned explicitly, while the postulates which are not satisfied are indicated by a minus sign.

The new examples are as follows (the class $K$ consisting of four elements, $1,2,3,4$, and the triads explicitly listed in each case being the only triads for which the relation $R$ is supposed to be true):

Ex. 41. 123, 134, 142, 143, 213, 214, 234, 241, 312, 321, 324, 341, $412,423,431,432$.

Ex. 42. 123, 124, 142, 241, 243, 321, 324, 342, 421, 432.

Ex. 43. 123, 143, 214, 243, 314, 321, 324, 412, 413, 423.

Ex, 44. 123, 143, 214, 231, 243, 312, 314, 412, 423, 431.

Ex. 45. 123, 132, 134, 142, 231, 241, 243, 321, 324, 342, 423, 431.

Ex. 46. 123, 124, 132, 134, 142, 213, 214, 231, 234, 241, 243, 312, $321,324,342,412,421,423,431,432$.

Ex. 47. 123, 142, 312, 314, 341, 342, 412, 423.

Ex. 48. 123, 321 .

Ex. 49. 123, 142, 324, 341.

Ex. 50. 123, 124, 312, 412, 431, 432.

Ex. 51. 123, 124, 231, 234, 241, 243, 341.

Ex. 52. 123, 231, 312, 412, 423, 431.

Ex. 53. 123, 134, 421, 423.

Ex. 54. 123, 124, 132, 134, 143, 213, 214, 231, 243, 312, 321, 324, $341,342,412,421,423,431$.

Ex. 55. 123, 132, 142, 143, 213, 231, 241, 243, 312, 321, 341, 342.

Ex. 56. 123, 143, 214, 243, 321, 324, 341, 342, 412, 423.

Ex. 57. 123, 124, 143, 243, 312, 341, 342, 412, 423.

LEMMAS ON NON-DEDUCIBILITY

We are now in position to prove 84 lemmas on non-deducibility, which, taken together, establish the fact that no other theorems on deducibility are possible besides the 116 theorems listed above. 
TABLe II'. List of 54 eXAMples OF PSEUdo-BETWEenNess

\begin{tabular}{|c|c|c|c|c|c|c|c|c|c|c|c|c|c|c|}
\hline Ex. & \multicolumn{13}{|c|}{ has properties } & \multirow{2}{*}{$\begin{array}{l}\text { Lemma in which example } \\
\text { is used }\end{array}$} \\
\hline $\begin{array}{l}\mathbf{A} \\
\mathbf{B} \\
\mathbf{C} \\
\mathbf{D}\end{array}$ & $\begin{array}{l}\overline{\mathbf{A}} \\
\mathbf{A} \\
\mathbf{A}\end{array}$ & $\frac{\mathrm{B}}{\mathrm{B}}$ & $\frac{\mathrm{C}}{\mathrm{C}}$ & $\begin{array}{l}\text { D } \\
\text { D } \\
\text { D } \\
-\end{array}$ & $\begin{array}{l}1 \\
1 \\
1 \\
1\end{array}$ & $\begin{array}{l}2 \\
2 \\
2 \\
2\end{array}$ & $\begin{array}{l}\mathbf{3} \\
\mathbf{3} \\
\mathbf{3} \\
\mathbf{3}\end{array}$ & $\begin{array}{l}4 \\
4 \\
4 \\
4\end{array}$ & $\begin{array}{l}\mathbf{5} \\
\mathbf{5} \\
\mathbf{5} \\
\mathbf{5}\end{array}$ & $\begin{array}{l}6 \\
6 \\
6 \\
6\end{array}$ & $\begin{array}{l}7 \\
7 \\
7 \\
7\end{array}$ & $\begin{array}{l}8 \\
8 \\
8 \\
8\end{array}$ & $\begin{array}{l}\mathbf{9} \\
\mathbf{9} \\
\mathbf{9} \\
\mathbf{9}\end{array}$ & \\
\hline 1 & $\mathbf{A}$ & B & $\mathrm{C}$ & $\mathrm{D}$ & - & 2 & 3 & - & $=$ & 6 & 7 & 8 & - & $1.1,4.1,5.2,9.2$ \\
\hline 2 & $\overline{\mathbf{A}}$ & $\mathbf{B}$ & $\mathrm{C}$ & D & - & - & - & 4 & $\mathbf{5}$ & 6 & 7 & 8 & - & $1.2,2.2,3.1,9.3$ \\
\hline $\mathbf{3}$ & $\mathbf{A}$ & $\mathbf{B}$ & $\mathrm{C}$ & $\mathrm{D}$ & 1 & - & 3 & 4 & - & - & - & - & - & $2.1,5.1,6.1,7.1,8.1,9.1$ \\
\hline 4 & $\mathbf{A}$ & B & $\mathrm{C}$ & D & - & - & - & 4 & 5 & - & 7 & - & - & $6.2,8.3$ \\
\hline 5 & $\overline{\mathrm{A}}$ & B & $\mathrm{C}$ & $\mathrm{D}$ & - & - & - & - & - & 6 & 7 & - & - & 8.2 \\
\hline 6 & $\mathbf{A}$ & - & $\mathrm{C}$ & $\mathrm{D}$ & - & 2 & 3 & 4 & 5 & 6 & 7 & 8 & - & 1.4 \\
\hline 7 & $\overline{\mathbf{A}}$ & - & $\mathrm{C}$ & $\mathrm{D}$ & 1 & - & 3 & - & - & 6 & - & - & - & $2.4,7.2,8.6$ \\
\hline 8 & $\overline{\mathbf{A}}$ & - & $\mathrm{C}$ & D & $\overline{1}$ & - & - & 4 & 5 & 6 & 7 & 8 & - & $2.5,3.5$ \\
\hline 9 & A & - & C & D & - & 2 & - & 4 & 5 & - & 7 & - & - & $3.6,6.8,8.5$ \\
\hline 10 & $\mathbf{A}$ & -- & $\mathrm{C}$ & D & 1 & 2 & 3 & - & - & 6 & 7 & 8 & - & $4.4,5.5$ \\
\hline 11 & $\mathrm{~A}$ & - & $\mathrm{C}$ & D & 1 & 2 & 3 & 4 & 5 & - & - & - & 9 & $6.7,7.3,8.4$ \\
\hline 12 & $\mathbf{A}$ & B & - & D & - & 2 & 3 & 4 & 5 & 6 & 7 & 8 & 9 & 1.3 \\
\hline 13 & $\vec{A}$ & B & - & D & 1 & - & 3 & 4 & 5 & 6 & 7 & 8 & 9 & 2.3 \\
\hline 14 & $\mathbf{A}$ & B & - & $\mathrm{D}$ & 1 & - & - & 4 & 5 & 6 & 7 & 8 & - & $3.2,9.5$ \\
\hline 15 & $\bar{A}$ & B & - & D & 二 & 2 & - & 4 & 5 & 6 & 7 & 8 & - & $3.3,9.6$ \\
\hline 16 & A & B & - & D & 1 & - & 3 & - & 5 & 6 & - & 8 & 9 & $4.2,7.4$ \\
\hline 18 & $\mathbf{A}$ & B & - & D & 1 & - & 3 & 4 & - & 6 & - & 8 & - & $\mathbf{5 . 3}, 7.5$ \\
\hline 19 & $\overline{\mathbf{A}}$ & B & - & D & 1 & 2 & 3 & 4 & 5 & - & - & - & 9 & $6.3,7.6,8.7$ \\
\hline 20 & $\mathbf{A}$ & B & - & D & - & - & 3 & 4 & 5 & 6 & - & - & 9 & $7.7,8.8$ \\
\hline 21 & $\overrightarrow{\mathbf{A}}$ & B & - & D & - & - & - & 4 & - & 6 & 7 & 8 & - & 5.4 \\
\hline 23 & A & B & - & D & 1 & - & 3 & 4 & - & 6 & - & - & - & 8.10 \\
\hline 24 & $\mathbf{A}$ & B & - & D & - & - & - & 4 & 5 & - & 7 & 8 & - & 6.4 \\
\hline 26 & - & B & C & $\overline{\mathrm{D}}$ & 1 & - & 3 & 4 & 5 & 6 & 7 & 8 & 9 & 2.6 \\
\hline 28 & - & B & C & D & 1 & 2 & 3 & - & - & 6 & 7 & 8 & - & 4.5 \\
\hline 29 & - & B & $\mathrm{C}$ & D & 1 & 2 & 3 & - & 5 & 6 & - & 8 & 9 & 4.6 \\
\hline 30 & - & B & $\mathrm{C}$ & D & 1 & - & 3 & 4 & 5 & 6 & - & 8 & 9 & 7.8 \\
\hline 32 & - & B & $\mathrm{C}$ & D & - & 2 & 3 & 4 & 5 & - & 7 & 8 & - & 6.11 \\
\hline 33 & - & B & C & D & 1 & 2 & 3 & 4 & - & 6 & 7 & 8 & - & 5.6 \\
\hline 36 & - & B & $\mathrm{C}$ & D & 1 & - & 3 & 4 & 5 & 6 & - & - & 9 & 8.14 \\
\hline 37 & - & B & $\mathrm{C}$ & D & - & - & 3 & - & 5 & 6 & 7 & - & - & 4.8 \\
\hline 38 & - & B & - & D & 1 & - & 3 & - & 5 & 6 & 7 & 8 & 9 & 4.10 \\
\hline 39 & $\mathbf{A}$ & - & - & D & - & 2 & - & 4 & 5 & - & 7 & 8 & - & 6.9 \\
\hline 40 & A & - & - & D & 1 & - & 3 & - & 5 & 6 & - & - & 9 & 8.11 \\
\hline 41 & A & B & - & D & - & - & - & 4 & 5 & 6 & 7 & 8 & 9 & 3.4 \\
\hline 42 & $\mathbf{A}$ & - & - & D & - & 2 & - & 4 & 5 & - & - & - & 9 & \\
\hline 43 & - & B & C & D & 1 & - & - & - & 5 & 6 & 7 & - & 9 & 4.7 \\
\hline 44 & - & B & C & D & - & - & 3 & - & 5 & - & 7 & - & 9 & 4.9 \\
\hline 45 & A & B & - & D & - & - & - & 4 & 5 & - & 7 & - & 9 & 6.5 \\
\hline 46 & A & B & - & D & - & - & - & - & 5 & - & - & 8 & 9 & 6.6 \\
\hline 47 & - & B & - & D & - & 2 & 3 & 4 & 5 & - & 7 & 8 & 9 & 6.13 \\
\hline 48 & A & - & C & D & 1 & 2 & 3 & 4 & 5 & 6 & 7 & 8 & - & 9.7 \\
\hline 49 & - & B & $\mathrm{C}$ & D & 1 & 2 & 3 & 4 & 5 & 6 & & 8 & - & 9.8 \\
\hline 50 & - & B & $\mathrm{C}$ & D & - & 2 & 3 & 4 & 5 & 6 & 7 & 8 & 9 & 1.5 \\
\hline 51 & - & B & C & D & 1 & 2 & - & 4 & 5 & 6 & 7 & 8 & 9 & \\
\hline 52 & - & B & $\mathrm{C}$ & D & - & 2 & 3 & 4 & 5 & - & 7 & - & 9 & $6.12,8.12$ \\
\hline 53 & - & B & $\mathrm{C}$ & $\mathrm{D}$ & 1 & 2 & 3 & 4 & 5 & - & - & - & 9 & $6.10,7.9,8.15$ \\
\hline 54 & $\mathbf{A}$ & B & - & D & - & 二 & - & 4 & 5 & 6 & 7 & -. & 9 & \\
\hline 55 & A & B & - & D & 1 & -- & 3 & 4 & - & 6 & - & 8 & - & 9.4 \\
\hline 56 & $\mathbf{A}$ & B & - & D & - & $\ldots$ & - & - & 5 & 6 & 7 & - & 9 & 4.3 \\
\hline 57 & - & $\mathrm{B}$ & $\mathrm{C}$ & D & - & - & 3 & 4 & 5 & 6 & 7 & - & 9 & 8.13 \\
\hline
\end{tabular}


TABLE III'. 84 LEMMAS ON NON-DEDUCIBILITY

\begin{tabular}{|c|c|c|c|}
\hline $\begin{array}{l}\text { Lem- } \\
\text { ma }\end{array}$ & $\begin{array}{l}\text { Post- } \\
\text { ulate }\end{array}$ & is not deducible from & $\begin{array}{l}\text { Proof } \\
\text { by } \\
\text { Ex. }\end{array}$ \\
\hline $\begin{array}{l}\text { A.1 } \\
\text { B.1 } \\
\text { C.1 } \\
\text { D.1 }\end{array}$ & $\begin{array}{l}\mathbf{A} \\
\mathbf{B} \\
\mathbf{C} \\
\mathbf{D}\end{array}$ & 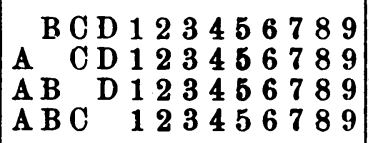 & $\begin{array}{l}\mathbf{A} \\
\mathbf{B} \\
\mathbf{C} \\
\mathbf{D}\end{array}$ \\
\hline $\begin{array}{l}1.1 \\
1.2 \\
1.3 \\
1.4 \\
1.5\end{array}$ & $\begin{array}{l}1 \\
1 \\
1 \\
1 \\
1\end{array}$ & 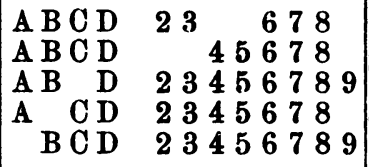 & $\begin{array}{c}1 \\
2 \\
12 \\
6 \\
50\end{array}$ \\
\hline $\begin{array}{l}2.1 \\
2.2 \\
2.3 \\
2.4 \\
2.5 \\
2.6\end{array}$ & $\begin{array}{l}2 \\
2 \\
2 \\
2 \\
2 \\
2\end{array}$ & 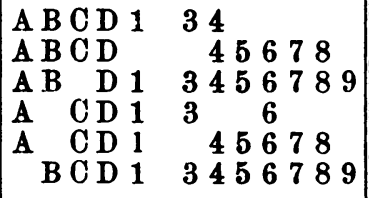 & $\begin{array}{c}3 \\
2 \\
13 \\
7 \\
8 \\
26\end{array}$ \\
\hline $\begin{array}{l}3.1 \\
3.2 \\
3.3 \\
3.4 \\
3.5 \\
3.6 \\
3.7 \\
3.8\end{array}$ & $\begin{array}{l}\mathbf{3} \\
\mathbf{3} \\
\mathbf{3} \\
\mathbf{3} \\
\mathbf{3} \\
\mathbf{3} \\
\mathbf{3} \\
\mathbf{3}\end{array}$ & 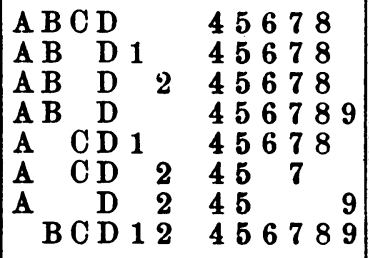 & $\begin{array}{c}2 \\
14 \\
15 \\
41 \\
8 \\
9 \\
42 \\
51\end{array}$ \\
\hline \begin{tabular}{l|}
4.1 \\
4.2 \\
4.3 \\
4.4 \\
4.5 \\
4.6 \\
4.7 \\
4.8 \\
4.9 \\
4.10
\end{tabular} & $\begin{array}{l}4 \\
4 \\
4 \\
4 \\
4 \\
4 \\
4 \\
4 \\
4 \\
4\end{array}$ & 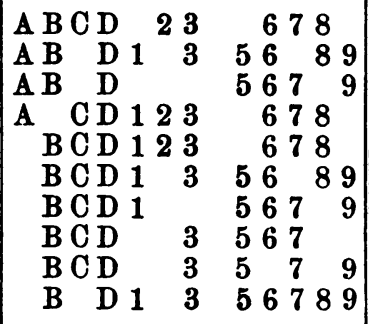 & $\begin{array}{l}1 \\
16 \\
56 \\
10 \\
28 \\
29 \\
43 \\
37 \\
44 \\
38\end{array}$ \\
\hline $\begin{array}{l}5.1 \\
5.2 \\
5.3 \\
5.4 \\
5.5 \\
5.6\end{array}$ & $\begin{array}{l}\mathbf{5} \\
\mathbf{5} \\
\mathbf{5} \\
\mathbf{5} \\
\mathbf{5} \\
\mathbf{5}\end{array}$ & 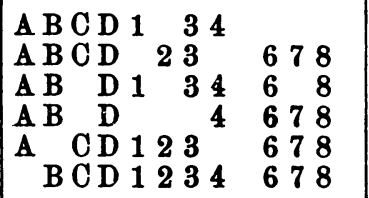 & $\begin{array}{c}3 \\
1 \\
18 \\
21 \\
10 \\
33\end{array}$ \\
\hline
\end{tabular}

\begin{tabular}{|c|c|c|c|}
\hline $\begin{array}{l}\text { Lem- } \\
\mathrm{ma}\end{array}$ & $\left|\begin{array}{l}\text { Post- } \\
\text { ulate }\end{array}\right|$ & is not deducible from & $\begin{array}{c}\text { Proof } \\
\text { by } \\
\text { Ex. }\end{array}$ \\
\hline $\begin{array}{l}6.1 \\
6.2 \\
6.3 \\
6.4 \\
6.5 \\
6.6 \\
6.7 \\
6.8 \\
6.9 \\
6.10 \\
6.11 \\
6.12 \\
6.13\end{array}$ & $\begin{array}{l}6 \\
6 \\
6 \\
6 \\
6 \\
6 \\
6 \\
6 \\
6 \\
6 \\
6 \\
6 \\
6 \\
6\end{array}$ & 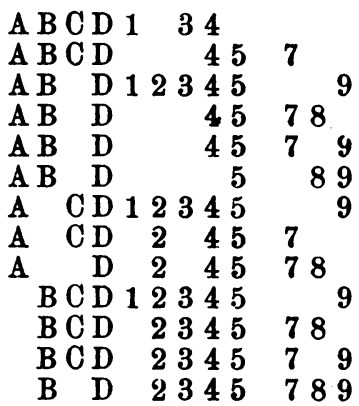 & $\begin{array}{c}3 \\
4 \\
19 \\
24 \\
15 \\
46 \\
11 \\
9 \\
39 \\
53 \\
32 \\
52 \\
47\end{array}$ \\
\hline $\begin{array}{l}7.1 \\
7.2 \\
7.3 \\
7.4 \\
7.5 \\
7.6 \\
7.7 \\
7.8 \\
7.9\end{array}$ & $\begin{array}{l}7 \\
7 \\
7 \\
7 \\
7 \\
7 \\
7 \\
7 \\
7 \\
7\end{array}$ & 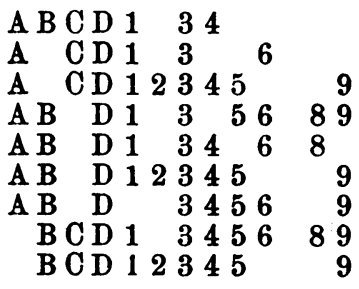 & $\begin{array}{l}3 \\
7 \\
11 \\
16 \\
18 \\
19 \\
20 \\
30 \\
53\end{array}$ \\
\hline $\begin{array}{l}8.1 \\
8.2 \\
8.3 \\
8.4 \\
8.5 \\
8.6 \\
8.7 \\
8.8 \\
8.9 \\
8.10 \\
8.11 \\
8.12 \\
8.13 \\
8.14 \\
8.15\end{array}$ & $\begin{array}{l}8 \\
8 \\
8 \\
8 \\
8 \\
8 \\
8 \\
8 \\
8 \\
8 \\
8 \\
8 \\
8 \\
8 \\
8\end{array}$ & 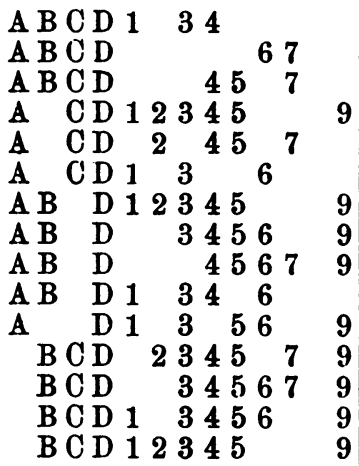 & $\begin{array}{c}3 \\
5 \\
4 \\
11 \\
9 \\
7 \\
19 \\
20 \\
54 \\
23 \\
40 \\
52 \\
57 \\
36 \\
53\end{array}$ \\
\hline $\begin{array}{l}9.1 \\
9.2 \\
9.3 \\
9.4 \\
9.5 \\
9.6 \\
9.7 \\
9.8\end{array}$ & $\begin{array}{l}\mathbf{9} \\
\mathbf{9} \\
\mathbf{9} \\
\mathbf{9} \\
\mathbf{9} \\
\mathbf{9} \\
\mathbf{9} \\
\mathbf{9}\end{array}$ & 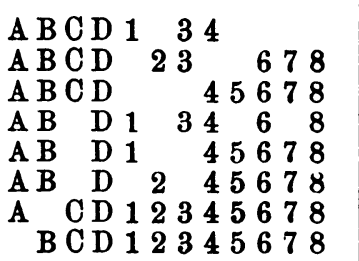 & $\begin{array}{l}3 \\
1 \\
2 \\
55 \\
14 \\
15 \\
48 \\
49\end{array}$ \\
\hline
\end{tabular}


Many of these lemmas were given in the earlier paper; but the new lemmas made necessary by the introduction of postulate 9 so often include certain of the old lemmas, that it is convenient to write out the whole list afresh, using a decimal notation instead of the letters of the alphabet, to avoid all possible confusion. This is done in Table $\mathrm{II}^{\prime}$, above.

It will be noticed that postulate $D$ plays a peculiar rôle. Although it is strictly independent and therefore cannot be omitted, yet it is not used in proving any of the theorems on deducibility, and it may always be made to hold or fail without affecting the holding or failing of any other postulate. It may therefore be called not only independent but altogether "detached".

Complete indePendence of postulates A, B, C, D, 9

To establish the complete independence* of the five postulates A, B, C, $D, 9$, we exhibit $2^{5}=32$ examples, which we number $000-031$ inclusive, in Table IV. In this table, a plus sign $(+)$ indicates that a postulate is satisfied, a minus sign (-) that it fails.

TABle IV. List of 32 eXamples for postulates A, B, C, D, 9

\begin{tabular}{|c|ccccc|}
\hline Ex. & A & B & C & D & 9 \\
\hline 000 & + & + & + & + & + \\
\hline 001 & - & + & + & + & + \\
002 & + & - & + & + & + \\
003 & + & + & - & + & + \\
004 & + & + & + & - & + \\
005 & + & + & + & + & - \\
\hline 006 & - & - & + & + & + \\
007 & - & + & - & + & + \\
008 & - & + & + & - & + \\
009 & - & + & + & + & - \\
010 & + & - & - & + & + \\
011 & + & - & + & - & + \\
012 & + & - & + & + & - \\
013 & + & + & - & - & + \\
014 & + & + & - & + & - \\
015 & + & + & + & - & - \\
\hline
\end{tabular}

\begin{tabular}{|c|ccccc|}
\hline Ex. & A & B & C & D & 9 \\
\hline 016 & - & - & - & + & + \\
017 & - & - & + & - & + \\
018 & - & - & + & + & - \\
019 & - & + & - & - & + \\
020 & - & + & - & + & - \\
021 & - & + & + & - & - \\
022 & + & - & - & - & + \\
023 & + & - & - & + & - \\
024 & + & - & + & - & - \\
025 & + & + & - & - & - \\
\hline 026 & - & - & - & - & + \\
027 & - & - & - & + & - \\
028 & - & - & + & - & - \\
029 & - & + & - & - & - \\
030 & + & - & - & - & - \\
\hline 031 & - & - & - & - & - \\
\hline
\end{tabular}

Example 000 shows that the five postulates are consistent.

Examples 001-005 show that the five postulates are independent in the ordinary sense; that is, no one of them is deducible from the other four.

*.H. Moore, Introduction to a form of general analysis, New Haven Colloquium, 1906, published by the Yale University Press, New Haven, 1910; p. 82. 
Examples 001-005 may be called "near-betweenness" systems, since they possess all but one of the five properties of betweenness. Examples 006-015 fail on two postulates; examples 016-025 fail on three, and examples $026-030$ on four; while example 031 fails to have any one of the properties characteristic of betweenness.

Ex. 000. 123, 124, 134, 234, 321, 421, 431, 432.

Ex. 001. 123, 124, 134, 234.

Ex. $002.123,124,321,421$.

Ex. 003. 123, 124, 134, 234, 321, 324, 421, 423, 431, 432.

Ex. 004. 123, 124, 134, 234, 321, 421, 431, 432; 444.

Ex. 005. 123, 143, 214, 234, 321, 341, 412, 432.

Ex. $006.123,124$.

Ex. 007. 123, 124, 324, 341, 342.

Ex. 008. 123, 124, 134, 234; 444.

Ex. 009. 123, 142, 324, 341.

Ex. 010. 123, 124, 142, 143, 241, 321, 341, 421.

Ex. 011. 123, 124, 321, 421; 444.

Ex. 012 . 123, 234, 321, 432.

Ex. 013 . 123, 124, 134, 234, 321, 324, 421, 423, 431, $432 ; 444$.

Ex. 014. 123, 214, 243, 314, 321, 324, 342, 412, 413, 423.

Ex. 015. 123, 143, 214, 234, 321, 341, 412, 432; 444.

Ex. 016. 123, 124, 132, 134.

Ex. 018. 123, 241.

Ex. 020. 123, 124, 134, 234, 243.

Ex. 023. 123, 124, 142, 241, 321, 421.

Exs. 017, 019, 021, 022, 024, 025. Same as Exs. 006, 007, 009, 010, 012,014 , with 444 added.

Ex. $026.123,124,132,134 ; 444$.

Ex. 027. 123, 124, 132.

Exs. 028, 029, 030. Same as Exs. 018, 020, 023, with 444 added.

Ex. 031. 123, 213, 234, 243, 423; 444.

This last system (Ex. 031) will be found to violate all the thirteen postulates of our basic list; it is therefore as far removed as possible from a true betweenness system. 


\section{SigNIFICANCE OF THE NOTION OF COMPLETE INDEPENDENCE*}

The significance of the notion of complete independence derives from the fact that every postulate may be stated, at pleasure, in either the positive or the negative form, so that every postulate, $a$, should be regarded as a pair of coördinate propositions, $a$ and $\bar{a}$. Thus a set of three postulates, $(a, \bar{a}),(b, \bar{b})$, $(c, \bar{c})$, divides the universe of discourse into $2^{3}=8$ compartments, represented by the logical products, $a b c ; \bar{a} b c$, $a \bar{b} c, a b \bar{c} ; \bar{a} \bar{b} c, \bar{a} b \bar{c}, a \bar{b} \bar{c} ; \bar{a} \bar{b} \bar{c}$, in which the barred and

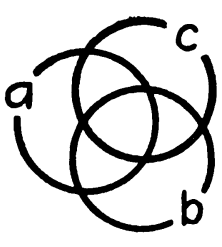
unbarred letters play precisely coördinate rôles.

If now there is no special relation between the postulates, all these compartments will be actually represented in the universe; it is only in the special case when some relation of implication among the propositions $a$, $\bar{a}, b, \bar{b}, c, \bar{c}$ holds true, that any one of these compartments will be empty.

For example, if $\bar{a} \bar{b} c$ is empty, then $\bar{a} c$ implies $b$ (and also $\bar{b} c$ implies $a$, and $\bar{a} \bar{b}$ implies $\bar{c}$ ); and, conversely, if any one of these three implications is valid, then the compartment $\bar{a} \bar{b} c$ will be empty. Similarly for each of the other compartments.

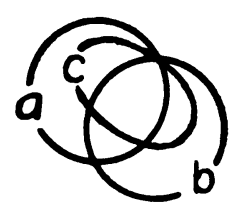

Hence Moore's criterion is a natural one: a set of $n$ postulates is "completely independent" when and only when no one of the $2^{n}$ compartments into which the postulates divide the universe is empty.

*Among the many papers on "complete independence" which have appeared in recent years may be mentioned the following:

R. D. Beetle, On the complete independence of Schimack's postulates for the arithmetic mean, Mathematische Annalen, vol. 76 (1915), pp. 444-446;

L. L. Dines, Complete existential theory of Shefjer's postulates for Boolean algebras, Bulletin of the American Mathematical Society, vol. 21 (1915), pp. 183-188;

E. V. Huntington, Complete existential theory of the postulates for serial order; and Complete existential theory of the postulates for well ordered sets, Bulletin of the American Mathematical Society, vol. 23 (1917), pp. 276-280 and pp. 280-282;

J.S. Taylor, Complete existential theory of Bernstein's set of four postulates for Boolean algebras, Annals of Mathematics, ser. 2, vol. 19 (1917), pp. 64-69; and Sheffer's set of five postulates for Boolean algebras in terms of the operation "rejection" made completely independent, Bulletin of the American Mathematical Society, vol. 26 (1920), pp. 449-454;

B. A. Bernstein, On the complete independence of Hurwitz's postulates for abelian groups and fields, Annals of Mathematics, ser. 2, vol. 23 (1922), pp. 313-316; and The complete existential theory of Hurwitz's postulates for abelian groups and fields, Bulletin of the American Mathematical Society, vol. 28 (1922), pp.397-399, and vol. 29 (1923), p. 33;

E. V. Huntington, Sets of completely independent postulates for cyclic order, Proceedings of the National Academy of Sciences, February, 1924;

W. E. Van de Walle, On the complete independence of the postulates for betweenness, in the present number of these Transactions. 
APPENDix, ON THE RELATION OF BETWEENNESS TO CYCLIC ORDER

The theory of betweenness (that is, the order of points along a straight line, without distinction of sense along the line), is closely related to the theory of cyclic order (that is, the order of points on a closed curve with a definite sense around the curve).*

Betweenness is characterized by the completely independent postulates $A$, B, C, D, 9; cyclic ordert by the completely independent postulates E, B, C, D, 9.

The postulates B, C, D, 9 hold true in both theories, while postulates A and $\mathrm{E}$ differ only by the interchange of two letters; thus:

Postulate A (for betweenness). If $A B C$, then $C B A$.

Postulate E (for cyclic order). If $A B C$, then $C A B$.

The following theorems may serve to bring out the contrast between the two theories.

Theorem on Betweenness. (From A, C, 9.) If $A, B$ are two distinct elements, and if $X, Y, Z$ are three other distinct elements, distinct from $A$ and $B$, and such that $X A B, A Y B, A B Z$; then $X Y Z$.

Proof. By 9, XAB.Y.?.XAY-YAB; and by 9, $A B Z$. I. . . $A B Y-Y B Z$. X A $Y$ B $Z$ But $Y A B$ and $A B Y$ conflict with $A Y B$, by $\mathrm{C}$ and $\mathrm{A}$; hence But $A Y X$ conflicts with $X A Y$, by $\mathrm{C}$ and $\mathrm{A}$; hence $X Y B$. Then by 9 , $X Y B . Z$. . .XYZ Z ZYB.

But $Z Y B$ conflicts with $Y B Z$, by $\mathrm{C}$ and $\mathrm{A}$; hence $X Y Z$.

Theorem on cyclic order. (From E, C, 9.) If $A, B, C$ are three distinct elements, such that $A B C$; and if $X, Y, Z$ are three other distinct elements, distinct from $A, B, C$ and such that $A X B, B Y C, C Z A$; then $X Y Z$.

* Besides (1) betweenness and (2) cyclic order, both of which are expressed in terms of a triadic relation, there are two other important types of order, namely: (3) serial order (that is, the order of points along a straight line with a definite sense along the line), which is expressed in terms of a dyadic relation; and (4) separation of point pairs (that is, the order of points on a closed curve without distinction of sense around the curve), which is expressed in terms of a tetradic relation. Sets of completely independent postulates for serial order are well known (loc. cit.); similar sets for the separation of point pairs will form the subject of a later paper.

† For the set E, B, C, D, 9, and two equivalent sets, E, B, C, D, 2 and E, B, C, D, 3, see E. V. Huntington, Sets of completely independent postulates for cyclic order, Proceedings of the National Academy of Sciences, February, 1924. 
Proof. By 9, $A B C . Y . \supset . A B Y-Y B C$, whence by $\mathrm{E}, Y A B-B C Y$. But $B C Y$ conflicts with $B Y C$, by $\mathrm{C}$. Hence $Y A B$. Then by 9, YAB.X.つ. YAX $-X A B$. But $X A B$ conflicts with $A X B$, by $\mathrm{E}$ and C. Hence $Y A X$, whence by $\mathrm{E}, A X Y$.

By $\mathrm{E}$ and 9, BCA.Y.つ. BCY $-Y C A$. But $B C Y$ con-

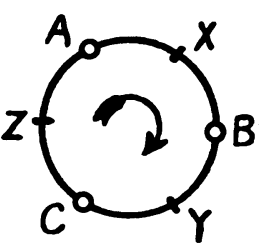

flicts with $B Y C$, by C. Hence $Y C A$. Then by $\mathrm{E}$ and $9, C A Y . Z$. つ.CAZ $Z A Y$. But $C A Z$ conflicts with $C Z A$, by $\mathrm{C}$. Hence $Z A Y$. Then by $\mathrm{E}$ and 9 , $A Y Z . X . \supset . A Y X-X Y Z$.

But $A Y X$ conflicts with $A X Y$, by C. Hence $X Y Z$.

The six postulates A, E, B, C, D, 9, taken together, would form, of course, an inconsistent set, since no system $(\mathrm{K}, \mathrm{R})$ has all these properties. It is interesting, however, to note the following "theorems of deducibility" among these six postulates.

Theorem 201. Proof of 9 from A, E, B.

To prove: $A B C \cdot X . \supset . A B X-X B C$. By $\mathrm{B}$, at least one of the six permutations of $A, B, X$ will be true; hence, by $\mathrm{A}$ and $\mathrm{E}$, all six will be true, so that $A B X$ will be true. Similarly, $X B C$ will be true.

Theorem 202. Proof of 9 from A, E, C.

To prove: $A B C . X . \supset . A B X-X B C$. Suppose 9 fails; that is, suppose $A B X$ and $X B C$ are both false while $A B C$ is true. Then by $\mathrm{A}$ and $\mathrm{E}$, we have $C B A$ and $C A B$, which conflict with each other, by C. Hence 9 must hold.

Theorem 203. Proof of B from not-A, E, C, and 9.

To prove: If $A, B, C$ are distinct, then at least one of the six permutations, $A B C, A C B, B A C, B C A, C A B, C B A$, is true; or, more briefly: If $A, B$, $C$ are distinct, then $P(A, B, C)$.*

Since postulate $A$ is violated, there must exist at least one true triad, say $X Y Z$.

Let $A$ be any element distinct from $X, Y, Z$.

By 9, $X Y Z . A . \supset . X Y A-A Y Z$. But if $X Y A$, then by $\mathrm{E}$ and 9 , $Y A X . Z . \supset . Y A Z-Z A X$; and if $A Y Z$, then by $\mathrm{E}$ and $9, Z A Y . X . \supset . Z A X-X A Y$.

Therefore $Y A Z-Z A X-X A Y$.

Case 1. If $Y A Z$, then by $\mathrm{E}$ and $9, A Z Y \cdot X . \supset . A Z X-X Z Y$; and by $\mathrm{E}$ and $9, Z Y A . X . \supset . Z Y X-X Y A$. But $X Z Y$ and $Z Y X$ conflict with $X Y Z$, by $\mathrm{E}$ and $\mathrm{C}$; hence, in Case $1, A Z X$ and $X Y A$.

\footnotetext{
- For essential details of this proof, including the convenient notation $P(A, B, C)$, I am indebted to Mr. C. H. Langford.
} 
Case 2. If $Z A X$, then by $\mathrm{E}$ and $9, A X Z . Y . \supset . A X Y-Y X Z$; and by $\mathrm{E}$ and $9, X Z A . Y . \supset . X Z Y-Y Z A$. But $Y X Z$ and $X Z Y$ conflict with $X Y Z$, by $\mathrm{E}$ and $\mathrm{C}$; hence, in Case $2, A X Y$ and $Y Z A$.

Case 3. If $X A Y$, then by $\mathrm{E}$ and $9, A Y X . Z . \supset . A Y Z-Z Y X$; and by $\mathrm{E}$ and $9, Y X A . Z . \supset . Y X Z-Z X A$. But $Z Y X$ and $Y X Z$ conflict with $X Y Z$, by $\mathrm{E}$ and $\mathrm{C}$; hence, in Case $3, A Y Z$ and $Z X A$.

Therefore (making use of E), we have

$X Y Z . A . \supset .(A Z Y . A Z X . A X Y)-(A X Z . A X Y \cdot A Y Z)-(A Y X . A Y Z . A Z X)$, whence

$$
P(X, Y, Z) . A . \supset . P(A, Y, Z) . P(A, Z, X) . P(A, X, Y),
$$

where the notation $P(A, X, Y)$, for example, means that at least one of the six possible permutations of the three letters, $A, X, Y$, forms a true triad.

Now let $B$ be any element distinct from $X, Y, Z, A$. Then, by the same reasoning,

$$
P(A, Y, Z) . B \text {. כ. } P(B, Y, Z) . P(B, A, Z) . P(B, A, Y) \text {. }
$$

Finally, let $C$ be any element distinct from $X, I, Z, A, B$. Then

$$
P(B, A, Y) . C . \supset . P(C, A, Y) \cdot P(C, B, Y) . P(C, B, A) \text {. }
$$

This last result, $P(C, B, A)$, states that at least one of the permutations of the letters $C, B, A$ forms a true triad, which establishes the theorem.

Theorem 204. Proof of not-B from A, E, C.

To prove: that three elements, $A, B, C$, exist, such that all six permutations, $A B C, A C B, B A C, B C A, C A B, C B A$, are false.

If the system contains no true triad, then the theorem is clearly true. If the system contains any true triad, say $X Y Z$, then by $\mathrm{A}$ and $\mathrm{E}$, we have $Z Y X$ and $Z X Y$, which is impossible, by C. Hence the theorem is true.

We are now prepared to exhibit the "complete existential theory" of these six postulates A, E, B, C, D, 9. The six postulates divide the universe into $2^{6}=64$ compartments, some of which, however, will be "empty." Thus, the four theorems just proved show that examples of the types
A, E, B, $\overline{9}$;
A, E, C, $\overline{9}$;
A, E, B, C;
$\overline{\mathrm{A}}, \mathrm{E}, \overline{\mathrm{B}}, \mathrm{C}, 9$ 
are impossible, so that at least ten of the 64 compartments will be empty (see the list in Table $\mathrm{V}$ below). This list shows that all the remaining 54 examples actually exist, so that the "existential theory" is complete.

Table V. Examples for the six inconsistent postulates A, E, B, C, D, 9

\begin{tabular}{|c|cccccc|c|}
\hline Rec. & A & E & B & C & D & 9 & Ex. \\
\hline$(1)$ & + & + & + & + & + & + & - \\
$(2)$ & + & + & + & + & + & - & - \\
3 & + & + & + & - & + & + & 037 \\
$(4)$ & + & + & + & - & + & - & - \\
5 & + & + & - & + & + & + & 038 \\
$(6)$ & + & + & - & + & + & - & - \\
7 & + & - & - & + & + & 039 \\
8 & + & - & - & + & - & 040 \\
9 & + & - & + & + & + & 000 \\
10 & + & - & + & + & + & - & 005 \\
11 & + & - & - & - & + & 003 \\
12 & + & - & + & - & + & - & 014 \\
13 & + & - & - & + & + & + & 002 \\
14 & + & - & - & + & + & - & 012 \\
15 & + & - & - & - & + & 010 \\
16 & + & - & - & - & + & - & 023 \\
17 & - & + & + & + & + & 033 \\
18 & - & + & + & + & - & 034 \\
\hline
\end{tabular}

\begin{tabular}{|c|cccccc|c|}
\hline Rec. & A & E & B & C & D & 9 & Ex. \\
\hline 19 & - & + & + & - & + & + & 035 \\
20 & - & + & + & - & + & - & 036 \\
$(21)$ & - & + & - & + & + & + & - \\
22 & - & + & - & + & + & - & 041 \\
23 & - & + & - & - & + & + & 043 \\
24 & - & + & - & - & + & - & 042 \\
25 & - & - & + & + & + & + & 001 \\
26 & - & - & + & + & + & - & 009 \\
27 & - & - & + & - & + & + & 007 \\
28 & - & - & + & - & + & - & 020 \\
29 & - & - & - & + & + & + & 006 \\
30 & - & - & - & + & + & - & 018 \\
31 & - & - & - & - & + & + & 016 \\
32 & - & - & - & - & + & - & 027 \\
\hline Records 33-64 are the same as Records \\
$1-32$ with D + changed to D-, and the \\
letter "d" added to each example-number \\
(in so far as these numbers exist). \\
\hline
\end{tabular}

The requisite examples, not already listed under Table IV, are as follows:

Ex. 033. 123, 231, 312; 124, 241, 412; 134, 341, 413; 234, 342, 423. Ex. 034. 123, 231, 312; 214, 142, 421; 134, 341, 413; 432, 324, 243. Ex. 035. 123, 231, 312; 312, 213, 132; 421, 214, 142; 431, 314, 143; $234,342,423 ; 432,324,243$.

Ex. 036. 123, 231, 312; 214, 142, 421; 134, 341, 413; 432, 324, 243 ; 321, 132, 213.

Ex. 037. All the twenty-four possible triads are true.

Ex. 038. No triads true.

Ex. 039. 123, 231, 312; 321, 132, 213; 124, 241, 412; 421, 214, 142 ; $234,342,423 ; 432,324,243$.

Ex. 040. 123, 231, 312; 321, 213, 132; 124, 241, 412; 421, 214, 142. Ex. 041. 123, 231, 312; 214, 142, 421.

Ex. 042. 123, 231, 312; 321, 213, 132; 214, 142, 421.

Ex. 043 Here the class $K$ consists of 5 elements, 1, 2, 3, 4, 5; all the sixty possible triads are true except the following: 321, 213, 132; $345,453,534 ; 543,435,354$.

Exs.033d, 034d, etc., are the same as Exs. 033, 034, etc., with the addition of the triad 444 (so as to violate postulate $D$ ). 
Finally, the inter-relations between the six postulates A, E, B, C, D, 9 may be shown diagrammatically as in the accompanying figure.* In this diagram, a zero in any compartment indicates that no example having the properties belonging to that compartment exists. For instance, the fact that no example of the type A, E, B, C, D, 9 exists, shows that the postulates are inconsistent.
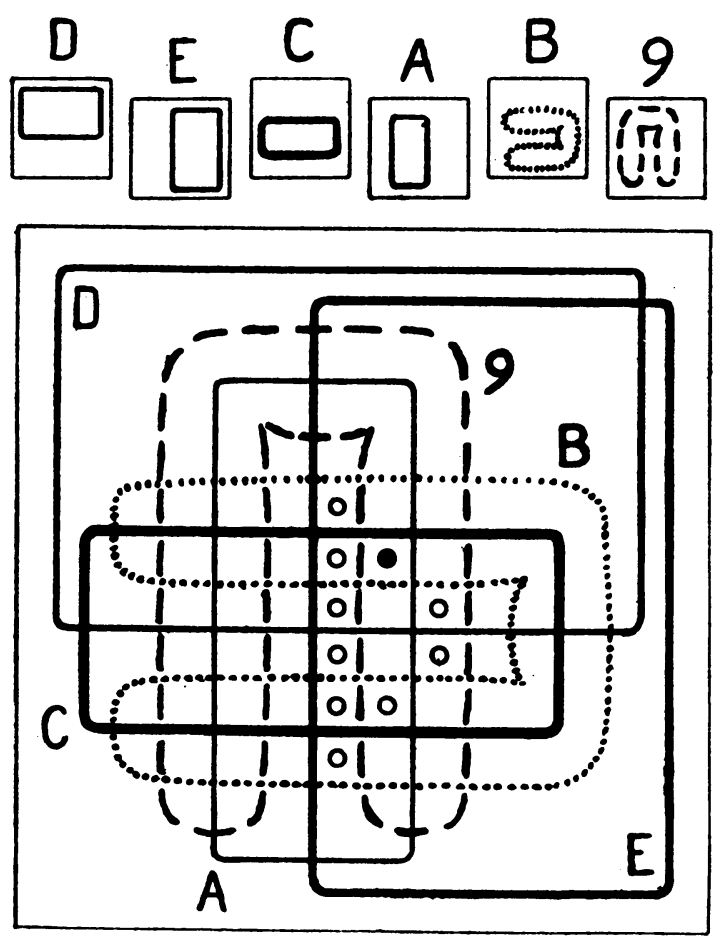

- This form of diagram, the possibility of which was vaguely suggested by Venn in 1881, is believed to be an improvement over those in common use. See

John Venn, On the diagrammatic and mechanical representation of propositions and reasonings, Philosophical Magazine, ser. 5, vol. 10 (July, 1880), pp.1-18; or his Symbolic Logic, 1st edition, 1881, p. 108, 2 d edition, 1894, p. 118 (with extensive historical notes);

H. Marquand, On logical diagrams for $n$ terms, Philosophical Magazine, ser. 5, vol. 12 (October, 1881), pp. 266-270;

C. L. Dodgson ["Lewis Carroll"], Symbolic Logic, London, 1896, known to me only through a citation by C. I. Lewis;

W. J. Newlin, $A$ new logical diagram, Journal of Philosophy, Psychology, and Scientific Methods, vol. 3 (1906), pp. 539-545;

W. E. Hocking, Two extensions of the use of graphs in elementary logic, University of California Publications in Philosophy, vol. 2 (1909), pp. 31-44; and

C. I. Lewis, A Survey of Symbolic Logic, University of Calif ornia Press, 1918, p. 1 S0.

HARVARD UNIVERSITY,

Cambridge, Mass. 